\title{
A VUELTAS CON LA INSOLVENCIA DE LOS ENTES PÚBLICOS Y LA VÍA CONCURSAL (EL CASO DE PUERTO RICO)
}

\author{
ISABEL FERNÁNDEZ TORRES ${ }^{1}$ \\ Universidad Complutense de Madrid/IDEIR \\ isfernan@ucm.es
}

Cómo citar/Citation

Fernández Torres, I. (2017).

A vueltas con la insolvencia de los entes públicos y la vía concursal (el caso de Puerto Rico).

Revista de Administración Pública, 204, 375-389.

doi: https://doi.org/10.18042/cepc/rap.204.13

\section{Resumen}

La crisis que atraviesa Puerto Rico no es solo una crisis económico-financiera, sino que hunde sus raíces en un problema político, el de su propia configuración como Estado Libre Asociado. Una situación cuasi colonial que le impide, por un lado, acogerse a la normativa de «quiebras» de los Estados Unidos, prevista para las municipalidades, pero también aprobar su propia normativa para afrontar la insolvencia de sus entes públicos. Una deuda de más de setenta y cuatro millones de dólares, cuya reestructuración se revela imposible, y más tras el huracán María, que está llevando a la Administración Trump a tener que plantearse una condonación — siquiera parcial— de la deuda. Pero, más allá de eso, habrá que reflexionar sobre sus soluciones a futuro, porque la crisis de Puerto Rico es una crisis estructural que requiere de la implementación de medidas estructurales.

1 Profesora titular de Derecho Mercantil. Realizado en el marco del proyecto «Unión Europea en el contexto de los tratados de nueva generación: entre la reforma institucional y la protección social» (DER2O16-76986-P). La revisión de este trabajo se ha realizado a finales de septiembre de 2017. La situación social, económica y política de la isla tras el huracán María es convulsa y puede llevar a muchos cambios en el futuro inmediato. 


\section{Palabras clave}

Estado; municipalidad; entes públicos; insolvencia; concurso; Estado Libre Asociado.

\section{Abstract}

The crisis Puerto Rico is going through is not only an economic-financial crisis, but it is rooted in a political problem that is that of its own configuration as a Commonwealth. A quasi-colonial situation that prevents it from, on the one hand, availing itself of the US bankruptcy regulations foreseen for municipalities but also from approving its own regulations to face the insolvency of its public entities. A debt of more than 74,000 million dollars whose restructuring is proving impossible and even more so after Hurricane Maria, which is leading the Trump Administration to consider cancelling — even partial — the debt. But beyond that, we will have to reflect on its future solutions because the crisis in Puerto Rico is a structural crisis that requires the implementation of structural measures.

\section{Keywords}

Municipality; State; insolvency; Bankruptcy; Commonwealth. 


\section{SUMARIO}

I. INTRODUCCIÓN. II. LA FACULTAD DE PUERTO RICO DE LEGISLAR: LA RECOVERY ACT. III. LA JUDICIALIZACIÓN DEL PROCESO. IV. SOLUCIONES A LA SITUACIÓN DE PUERTO RICO: UNA PERSPECTIVA COMPARADA.

\section{INTRODUCCIÓN}

En ocasiones anteriores me he referido a las causas que están en el origen de la crisis de Puerto Rico, así como a las particularidades del derecho norteamericano de quiebras y sus peculiaridades respecto del derecho europeo ${ }^{2}$. No pretendo en este momento volver sobre ello, sino, de alguna manera, partir de cuanto he señalado para centrar mi atención en las novedades que se han ido produciendo en este ámbito desde entonces. En especial, me ocuparé de la judicialización de la insolvencia de Puerto Rico. Todo lo cual irá acompañado de una breve referencia tanto a las posibles soluciones cuanto a las iniciativas que se están poniendo en marcha en el ámbito de la Unión Europea en materia de reestructuración de deuda.

Como podemos recordar, Puerto Rico tiene vedado el recurso al procedimiento de quiebras recogido en el capítulo 9 del Bankruptcy Code dedicado al «adjustment of debts of a municipality» ${ }^{3}$ desde que en 1984 , como consecuencia de unas correcciones de carácter técnico y en virtud de la nueva definición introducida sobre el concepto de «Estado», se excluyera — sin razones o explicación alguna aparente- a Puerto Rico —en tanto Estado Libre Asociado- de su ámbito de aplicación ${ }^{4}$. De hecho, tal es la confusión que a

2 I. Fernández Torres (20 I 5), «El Estado Libre Asociado de Puerto Rico necesita declararse en "Bancarrota”", en Revista Administración Pública, 198, págs. 369-382, http:// eprints.ucm.es/37326/

3 Sobre el procedimiento concursal aplicable a las municipalidades, véase I. Fernández Torres (20 I 5), El concurso de las entidades del sector público, Cizur Menor: Aranzadi.

4 Bankruptcy Amendments and Federal Judgeship Act (1984), Pub. L. No. 98-353, 98 Stat. 333 [enacting 11 U.S.C. $\$ 101$ (52)]. La problemática radicaba en parte en la definición de los conceptos governmental unit, State y Commonwealth. Su dife- 
Puerto Rico se le aplica todo el Bankruptcy Code a excepción del capítulo 9. La cuestión no planteó ningún problema durante los ańos de expansión o crecimiento económico. Sin embargo, la recesión y, en consecuencia, la situación de crisis iniciada en $2008^{5}$, llevaron a que los fondos de cobertura dejaran de comprar la deuda, lo que derivó no solo en una crisis fiscal y de sostenibilidad del Estado en servicios esenciales y básicos, sino también a la judicialización del proceso, que ha reabierto el debate sobre la propia configuración y estatus jurídico de la isla. Un debate que, tras conocerse la decisión en el asunto Sánchez Valle ${ }^{6}$, se reabrió con toda su crudeza. La ya muy conocida Sentencia Sánchez Valle vino a marcar el camino que habría de seguirse en las futuras decisiones que el Tribunal Supremo de los Estados Unidos pudiera adoptar en relación con Puerto Rico y su soberanía. En dicho asunto, el Tribunal Supremo de Estados Unidos aceptó revisar la decisión del Tribunal Supremo de Puerto Rico a los efectos de que la eliminación de cargos criminales en el ámbito estatal a una persona previamente convicta por la misma ofensa en la esfera federal implica una violación de la cláusula sobre la exposición dual de la Quinta Enmienda de la Constitución de los Estados Unidos. El Tribunal Supremo de Puerto Rico entendió, en virtud de la doctrina de la soberanía dual, que los Estados tienen reconocido un grado de soberanía que les permite encausar a una persona que ha sido previamente encausada en el ámbito federal (y viceversa), y ello por cuanto que, al tratarse de un soberano distinto el que castiga la ofensa, no puede entenderse que se produzca una violación de la Quinta Enmienda.

rente conceptualización se interpretó como indicativa de un intento de aplicar el Código de 1978 solo a los cincuenta Estados de la Unión, si bien la jurisprudencia se movía en dirección opuesta: Calero-Toledo v. Pearson Yacht Leasing Co., 416 US. 663, 675 (1974); Americana of Puerto Rico, Inc. v. Kaplus, 368 F. 2d 431, 438 (3d Cir. 1966); United States v. Laboy-Torres, 553 F. 3d 715, 722 (3d Cir. 2009); J. O’Connor (2000), "Jusino Merca- do v. Commonwealth of Puerto Rico», 214 F. 3d 34, pág. 42.

5 Se estima que el total de la deuda pública de Puerto Rico es de alrededor de 72000 millones de dólares. De ese total, las corporaciones públicas son responsables de alrededor un $68 \%$, incluyendo casi 9000 millones atribuibles a la Autoridad de la Energía Eléctrica (véase Financial Information and operating Data Report (Commomwealth of Puerto Rico), 6 de noviembre de 2015, http://www.gdb-pur.com/documents/CommonwealthReport11-06-15.pdf.).

6 Pueblo c. Sánchez Valle (2015), "Cert. Granted sub nom», Puerto Rico v. Sánchez Valle, 136, S. Ct. 28, 192 DPR 594. A este respecto, puede ser de interés consultar el informe: R. S. Garrett (2016), «Political Status of Puerto Rico: brief Background and recent developments for Congress», R44721, www.crs.gov. 
En efecto, en la sentencia se determina que la soberanía del Estado Libre Asociado no es la plena, característica de los países independientes, sino más limitada. Se trata de una soberanía condicionada, en definitiva, por la cláusula de Supremacía de la Constitución de los Estados Unidos, en virtud de la cual la Constitución y las Leyes Federales son ley suprema y se sitúan por encima de las leyes estatales ${ }^{7}$. Una limitación que se percibe con toda su crudeza cuando tratan de precisarse las competencias del Estado Libre Asociado a la hora de abordar su crisis.

Y es que, ante la imposibilidad de aplicar la normativa sobre quiebras de EE.UU., dos fueron las principales líneas de trabajo del Gobierno de Puerto Rico. Por un lado, para atender el problema de la insolvencia de sus municipalidades o subdivisiones políticas y en virtud del ejercicio responsable de sus poderes, Puerto Rico optó por aprobar una normativa propia que protegiera adecuadamente sus corporaciones públicas de los acreedores y que, al mismo tiempo, garantizara la continuidad en la prestación de los servicios públicos. Así, con la promulgación de la conocida como «Ley Criolla», el Gobierno no solo ejerció sus poderes, sino que lo hizo de forma responsable. Sin embargo, esta iniciativa fue impugnada ante los tribunales por algunos de los acreedores al entenderse que Puerto Rico carecía de soberanía para ello. Por otro lado, el Gobierno inició una intensa campańa para tratar de que la normativa federal sobre quiebras pudiera ser aplicada, en su integridad, a Puerto Rico.

Lamentablemente, la situación económico-financiera de la isla se fue deteriorando tanto que llevó a que se creara la Junta de Supervisión Fiscal en virtud de la Ley Puerto Rico Oversight, Management and Economic Stability Act de 2016 (PROMESA). La Junta está formada por siete miembros nombrados por el presidente de los Estados Unidos y un miembro nombrado por el gobernador de Puerto Rico.

Trataremos a continuación de comentar los hitos más importantes del proceso de judicialización y politización de la insolvencia de Puerto Rico, refiriéndonos asimismo a las nuevas iniciativas que se están produciendo en el marco del derecho comparado.

7 Algunos no han dudado en señalar la condición colonial de la isla de Puerto Rico, véase el análisis de Torruella, en la conferencia pronunciada ante el Colegio de Abogados de Puerto Rico el 10 de septiembre de 2016: http://www.elnuevodia.com/opinion/ columnas/puertoricoalbate-columna-2240568/. 


\section{LA FACULTAD DE PUERTO RICO DE LEGISLAR: LA RECOVERY ACT}

La situación de vacío legal llevó al Congreso del Estado Libre Asociado a aprobar la Ley 71, de 28 de junio de 2014, para el cumplimiento con las deudas y la recuperación de las Corporaciones Públicas, conocida coloquialmente como «ley de quiebra criolla» o Recovery $A c t^{8}$. Ley que debía aplicarse fundamentalmente al cumplimiento, repago y reestructuración de las deudas de las corporaciones públicas:

Principalmente de:

- autoridad de la energía eléctrica;

- autoridad de acueductos y alcantarillado;

- autoridad de carreteras y transportes.

La Recovery Act o ley criolla, como se la conoce del otro lado del Atlántico, contempla dos tipos de procedimientos:

- por un lado, el consensual de modificación de la deuda, que culmina con un programa de recuperación (capítulo 2);

- el procedimiento supervisado por el Tribunal, que culmina con un plan ordenado de cumplimiento de deudas (capítulo 3).

De esta manera, una corporación pública puede solicitar la aplicación tanto del capítulo 2 como del capítulo 3, o de ambos de forma consecutiva o concurrente. La Ley está diseñada en muchos aspectos para que refleje ciertas disposiciones clave del capítulo 11 del Código norteamericano y, tanto el Tribunal como las partes interesadas, deben revisar y considerar los precedentes existentes al amparo del título 11 en el momento de interpretar e implementar esta ley.

La norma se dirige solo a corporaciones públicas, quedando excluido tanto el propio Estado Libre Asociado como la deuda garantizada por el Estado, los setenta y ocho municipios, el Banco Central y otras instituciones expresamente contempladas por la Ley.

8 E. Sanjuán y Muñoz (2016), «La aplicación del capítulo 9 del Código de quiebras norteamericano al Estado Libre Asociado de Puerto Rico", en Revista Concursal y Paraconcursal, 24; S. J. Lubben (20I4), «Puerto Rico and the bankruptcy clause», https://papers.ssrn.com/sol3/papers.cfm?abstract_id=2514824; M. K. Piacentini (201 5), «Lights out for Puerto Rico's Restructuring Law», en Brook. L.Rev., 80, pág. 1677. 
El capítulo 2 regula un mecanismo para que una corporación pública adopte un programa de recuperación que vincule a todos los tenedores de deuda con el consentimiento de una súper mayoría de ellos. Consiste en cualquier combinación de modificaciones y enmiendas a los instrumentos de deuda afectados, siempre que estas se combinen con el compromiso de la corporación de estar sujeta al plan de reestructuración. Las enmiendas pueden incorporar elementos diversos: ajustes de tasas de interés, extensión del vencimiento, reducción del principal y otras revisiones a los instrumentos de deuda afectados.

El capítulo 3 atiende al problema de deuda de las corporaciones a través de una solución de carácter judicial, que exige los mismos requisitos que los capítulos 9 y 11 del título 11. Permite que las corporaciones públicas puedan aplazar el repago de su deuda, reducir el interés y el principal, según sea necesario, de modo que pueda seguir cumpliendo sus funciones públicas esenciales.

La Ley establece un sistema de reestructuración de la deuda más allá de lo que prevé el capítulo 9 del Código de Quiebras norteamericano, y se fundamenta precisamente en la inaplicabilidad de este. $\mathrm{Y}$ eso es precisamente lo que hace surgir la controversia que se ha llevado a los tribunales federales.

\section{LA JUDICIALIZACIÓN DEL PROCESO}

El grupo de inversores «Franklin y Blue Mountain Capital Management», tenedores de bonos emitidos por la Autoridad de Energía Eléctrica por importe de dos mil millones de dólares, demandó al Estado Libre Asociado de Puerto Rico y a algunos representantes del Gobierno, al entender que este no tenía capacidad para legislar en materia de quiebras, alegando que dicha competencia reside en el Congreso de los Estados Unidos. Así, llevaron la Recovery $A c t^{9}$ al Tribunal de Distrito, que dictó la Sentencia 6 de febrero de $2015^{10}$, declarando la inconstitucionalidad de la Ley 71 de 2014. En dicha sentencia, el juez Besosa señala que la citada normativa infringe la competencia normativa federal y, por tanto, entiende que no corresponde al Estado

9 Franklin California Tax-Free Trust v. Commonwealth of Puerto Rico, 805 F. 3d 322 (1st Cir. 2015), petition for cert. Granted, 84, U.S.L.W. 3100 (U.S., 4 diciembre de 2015) (No. 15-233).

Uno de los aspectos más problemáticos es el relativo al «automatic stay» recogido en la Ley de quiebra criolla en virtud del cual se paralizaban los procedimientos en los tribunales federales en tanto se resolvía la quiebra en la isla.

10 Franklin California Tax-Free Trust v. Puerto Rico, 85 F. Supp. 3d 577 (2015). 
Libre Asociado adoptar ningún tipo de medidas de reestructuración de la deuda pública al amparo de lo dispuesto en ella. La sentencia fue apelada y, el 6 de julio de 2015, el Tribunal de Apelaciones de Estados Unidos del Primer Circuito («Tribunal de Apelaciones») confirmó la decisión del Tribunal de Distrito de Estados Unidos para el Distrito de Puerto Rico ${ }^{11}$, decisiones que fueron posteriormente ratificadas por el Tribunal Supremo de Estados Unidos, en su Sentencia de 13 de junio de $2016^{12}$.

El fundamento de dichas decisiones radica en que la Constitución federal estadounidense recoge, en el art. I, sección $8^{a}$, entre las competencias del Congreso Federal la potestad de "establish [...] uniform Laws on the subject of Bankruptcies throughout the United States».

Las sentencias de Distrito y de Apelación, en su resolución, deberían haber tenido en cuenta - en opinión de algunos autores ${ }^{13}$ - el caso Puerto Rico Department of consumer Affairs c. Isla Pretoleum ${ }^{14}$, de 1988, un caso similar en cuanto a competencias legislativas ${ }^{15}$. Sin embargo, entendieron que

11 L. Cardona Figuera (2015), «La excepción sin explicación: Puerto Rico ante la crisis económica y el capítulo 9 de quiebras federal», en Revista de Estudios Críticos, 12, págs. 202 y ss.

12 http://www.justicia.pr.gov/wp-content/uploads/2016/06/Decisión-SupremoEEUU-6-13-2016.pdf .

13 J. L. Nieto Mingo (20 I 6), «La doctrina del campo ocupado y la facultad del gobierno de Puerto Rico para implementar soluciones a la situación de insolvencia de sus subdivisiones políticas», en Revista Jurídica UPR, 85, 3.

14 Puerto Rico Dep't of Consumer Affairs, 485 U.S. 495.

15 Los hechos del caso son relativamente sencillos. Una Ley de EE.UU. de los años sesenta del siglo XX prohibía que los estados reglamentaran los precios de la gasolina [Emergency Petroleum Allocation Act of 1973, Pub. L. 93-159, 87 Stat. 627 (1973)]. Dicha norma señala expresamente que, transcurrido un determinado plazo tras su entrada en vigor, expiraría de tal manera que mientras la Ley estuviese en vigor, los Estados (Puerto Rico incluido) no podría reglamentar los precios de la gasolina. Cuando la Ley expiró, el Departamento de Asuntos del Consumidor (DACO) comenzó a reglamentar los márgenes de ganancia de la gasolina, lo que llevó a que los mayoristas demandaran al DACO en la Corte de Distrito, argumentando que carecía de competencia para reglamentar los precios de la gasolina porque el Congreso había ocupado el campo. El argumento de las compañías gasolineras del momento era el siguiente: al expirar la Ley federal, la intención del Congreso era que no legislara nadie, sino que fuera el propio mercado el que, en virtud de la ley de oferta y demanda, fijara el precio de la gasolina. El asunto llegó hasta el Tribunal Supremo de EE.UU. y el juez Scalia señaló que el juicio acerca de la preemption realizado en instancias inferiores era erróneo. En su opinión, si el Congreso quería ocupar el campo, podía hacerlo. Concluye que, una vez el Congreso decide retirarse del campo, no puede haber preemption posible. 
el Congreso había «ocupado el campo» y que los Estados no podían legislar (preemption $)^{16}$, lo contrario de lo que el juez Scalia señaló en el asunto anteriormente indicado, en relación con el cual consideró que cuando una Ley se deroga sin ser sustituida por otra, no puede haber preemption. Y esto es lo mismo que pasó en 1984, cuando el Congreso se retiró del campo al enmendar el código de quiebras. Ante la ausencia de una ley que lo prohibiera, y ejerciendo su función, Puerto Rico legisló.

Especialmente interesante se revela el voto particular del juez R. Torruella del Valle (juez del Tribunal de Apelaciones para el Primer Circuito), quien seńala no solo la inconstitucionalidad de la Ley Criolla, sino que va más allá y la invoca también respecto de la reforma de 1984, que establece un trato dispar a la isla sin una base racional que lo justifique. Desde esta perspectiva, entiende que todo trato dispar debe estar debidamente justificado, ser racional y claro. Señala, además, que, con el criterio mantenido por la opinión mayoritaria, el Tribunal acaba reforzando la relación colonialista y de dependencia entre Puerto Rico y Estados Unidos.

Ante esta situación, el Gobierno planteó una cuestión de competencia (writ certiorari) a los efectos de que el Tribunal Federal se pronunciara sobre la capacidad legislativa de la isla defendiendo que, puesto que en 1984 se excluyó a Puerto Rico del capítulo 9, el campo para poder legislar en ese ámbito nunca fue desarrollado, por lo que la Recovery Act era válida.

En tanto el Tribunal Federal resolvía, y siguiendo la recomendación del Tribunal de Apelación, Puerto Rico presentó una enmienda al Código de Quiebras para poder aplicarlo: Proyecto de Ley «H.R. 870. To amend title 11 of the United States Code to treat Puerto Rico as a State for purposes of chapter 9 of such title relating to the adjustment of debts of municipalities» ${ }^{17}$, que propugna que Puerto Rico sea equiparado al resto de los Estados a los efectos de poder aplicar el capítulo 9 del Bankruptcy Code.

La propuesta legislativa no va en la línea de autorizar a todas las municipalidades a solicitar el concurso sin más, sino a permitir que el Estado de Puerto Rico adopte la decisión que estime más oportuna sobre qué municipalidades y en qué condiciones pueden presentar la solicitud. De hecho, conviene advertir que el ámbito de aplicación de la norma está bastante restringido en el sentido de que ni las ciudades ni el propio Estado Libre Asociado de Puerto Rico pueden someterse al procedimiento ${ }^{18}$.

16 Véase S. J. Lubben (20 I4).

17 https:/www.congress.gov/bill/114th-congress/house-bill/870/text?format=txt. A este respecto, puede ser interesante consultar el testimonio de J. A. E. Pottow (2015): https:// judiciary.house.gov/wp-content/uploads/2016/02/John-Pottow-Testimony.pdf

18 Para más detalles me remito a lo que ya expuse anteriormente (Fernández Torres, 201 5 ). 
Lamentablemente, el 13 de junio de 2016, el Tribunal Supremo falló en el sentido argumentado por los fondos de inversiones: la reforma de 1984 excluyó a Puerto Rico de la sección 109.2 c). Conviene destacar la opinión de la juez Sotomayor que señaló que, con la reforma de 1984, la intención del Congreso fue privar a Puerto Rico de la aplicación del capítulo 9. La decisión del Tribunal Supremo es clara: Puerto Rico no es un Estado a los efectos de poder autorizar a sus municipalidades acogerse al capítulo 9 pero tampoco es un Estado a los efectos de la prohibición del Congreso que impide a los Estados legislar sus propias leyes de quiebras.

El resultado todos lo conocemos, en junio de 2016 se aprueba la Ley para la supervisión, administración y estabilidad económica para Puerto Rico (PROMESA) ${ }^{19}$, que instaura, a partir del 1 de septiembre de 2016, la Junta de Control Fiscal, la cual se crea como una entidad dentro del Gobierno de Puerto Rico. Dicha Junta tiene como una de sus misiones fundamentales elaborar y aprobar un plan para el proceso de desarrollo, presentación, aprobación y certificación de los planes fiscales, así como asegurar la implementación de estos. Para ello tiene la potestad de requerir al Gobierno de Puerto Rico que incluya en dichos planes cualquier recomendación dada por la Junta de Supervisión, aun cuando esta pueda haber sido rechazada previamente por el Congreso de la isla o por el gobernador (sección 201 PROMESA). La aprobación de dichos planes, extremadamente duros, ha generado muchos rechazos en la isla, huelgas..., en definitiva, una fuerte tensión. Ante la imposibilidad de alcanzar un acuerdo con los acreedores que evite una avalancha de demandas, el gobernador ha recurrido al título III de PROMESA (un híbrido entre el capítulo 9 y el 11 del Código de quiebras norteamericano), que establece un proceso de reestructuración de la deuda supervisado por un tribunal y que paraliza cualesquiera litigios asociados a la deuda. Dicha petición se formuló los días 3 y 5 de mayo de 2017.

19 A. Austin (2016), "The Puerto Rico Oversight, management, and Economic Stability Act», PROMESA, H.R. 5278, S. 2328, CRS Report 44532, https://www.congress. gov/bill/114th-congress/house-bill/5278?q=\%7B\%22search $\% 22 \% 3 \mathrm{~A} \% 5 \mathrm{~B} \% 22 \mathrm{CR}$ S+Report $+44532 \% 2 \mathrm{C}+$ The + Puerto+Rico+Oversight $\% 2 \mathrm{C}+$ management $\% 2 \mathrm{C}+$ and + Economic+Stability+Act+\%28PROMESA\%2C+H.R.+5278\%2C+S.+2328\%29\% 22\%5D\%7D\&r=4 https://noticiasmicrojuris.files.wordpress.com/2017/02/ley-promesatraducida-al-espac3b1ol.pdf 


\section{SOLUCIONES A LA SITUACIÓN DE PUERTO RICO: UNA PERSPECTIVA COMPARADA}

¿Es una solución para Puerto Rico haber puesto en marcha el título III de PROMESA y proceder a la reestructuración de la deuda? La reestructuración de la deuda al amparo de PROMESA, al igual que sucede en EE.UU., no soluciona sus problemas porque, tal y como puso de manifiesto el Informe Krueger el problema de la isla es estructural, como lo son los de muchas corporaciones públicas; por ello, es preciso que las reformas que se emprendan sean también estructurales ${ }^{20}$. Es más, los planes elaborados al amparo de PROMESA parecen estar sumiendo a la isla en una profunda recesión ${ }^{21}$.

De hecho, algunos autores en EE. UU. vienen manifestándose en los últimos tiempos en contra de la aplicación del régimen concursal a las municipalidades, por diversos motivos. En primer lugar, qué duda cabe que el concurso tiene un coste reputacional y económico para la municipalidad muy elevado (los costes económicos derivan no solo del procedimiento per se, sino del hecho de que, al lesionarse la reputación de la municipalidad, su actividad económica puede verse dañada). Pero es que, además, se ha constatado que el derecho concursal aplicable a las municipalidades no soluciona ni modifica ninguna de las circunstancias que favorecieron la crisis de la municipalidad. El procedimiento concursal busca una reestructuración de las deudas y, aún cuando la renegociación se hace desde una posición de fuerza, la municipalidad verá su deuda reducida, pero en ningún caso condonada. Por el contrario, al no alterarse las bases que coadyuvan a esa situación de crisis, será fácil —y, de hecho, en la práctica, se ha planteado muchas veces - que, transcurrido un cierto plazo, la municipalidad deba acogerse de nuevo a un procedimiento concursal ${ }^{22}$. Y es que, como bien exponen algunos autores, los gastos, la estructura de ingresos y, en definitiva, todo el funcionamiento de la municipalidad se mantienen inalterados tras el concurso. Es por ello por lo que el procedimiento solo se revela adecuado cuando la insolvencia se produce como consecuencia de la concurrencia de un acontecimiento, digamos, inesperado, que altera sustancialmente la economía del ente municipal (un desastre natural, el pago de una indemnización...), y no lo es para resolver un problema de carácter financiero/fiscal endémico ${ }^{23}$. Y eso es lo que puede estar ocurriendo

20 J. V. González García, http://www.globalpoliticsandlaw.net/category/puerto-rico/.

21 https://www.project-syndicate.org/commentary/puerto-rico-debt-plan-deep-depression-by-joseph-e--stiglitz-and-martin-guzman-2017-02

22 O. Kimhi (20Iо), "Chapter $9 \mathrm{f}$ the Bankruptcy Code: a solution in search of a problem», en Yale Journal on Regulation, 27, págs. 351-359.

23 C. Gilette (20I 2), «What States can learn from Municipal Insolvency», en P. Conti-Brown y D. A. Skeel, Jr. (eds.), When States go broke, Cambridge University Press, pág. 99. 
en el momento en el que se cierra este trabajo. El huracán María ha dejado la isla de Puerto Rico devastada ${ }^{24}$ y el Congreso de EE. UU. ha adoptado un conjunto de medidas el 16 de noviembre de 2017 que solo podrá contribuir a asfixiar su economía: aranceles, incentivos fiscales para las entidades que se trasladen a territorio norteamericano. La recuperación plena de la economía de Puerto Rico pasa necesariamente por su "descolonización»" ${ }^{25} \mathrm{y}$, en consecuencia, por eliminar definitivamente su sujeción a la Jones Act y otras normas similares ${ }^{26}$.

Si el procedimiento concursal no se revela adecuado ${ }^{27}$, ¿qué soluciones pueden, pues, propugnarse? Estos autores abogan por un modelo de supervisión estatal proactivo, que opere no solo cuando la sociedad es insolvente, sino que sea el Estado quien controle las entidades de forma continua y, cuando aprecie signos de crisis, intervenga para — de forma preventiva - evitar que ello siga evolucionando. Esa supervisión activa implica la creación de un consejo que vigile la municipalidad y que sea el que, llegada la ocasión, prepare un plan de ajuste fundado, por un lado, en la reducción de costes y, por otro, en

24 https://www.project-syndicate.org/commentary/puerto-rico-hurricane-new-energygrid-by-simon-johnson-2017-09

https:/www.project-syndicate.org/commentary/how-puerto-rico-can-recover-byjose-antonio-ocampo-2016-06. Sobre la situación de dependencia e incluso colonial de la Isla véase "The International Place of Puerto Rico", APR 10, 2017, 130 Harv. L. Rev. 1656 https://harvardlawreview.org/2017/04/the-international-place-of-puertorico/

26 El senador John McCain presentó en julio de 2017 un proyecto de ley para revocar las restricciones de cabotaje de la Jones Act, https://aldia.microjuris.com/2017/07/19/senador-john-mccain-presenta-proyecto-de-ley-para-revocar-la-ley-jones/, proyecto de ley: https://noticiasmicrojuris.files.wordpress.com/2017/07/jones-act-repeal-7-13-17. pdf. Por el momento, y como consecuencia del huracán María, el gobierno de Trump ha suspendido temporalmente la aplicación de las leyes de cabotaje a Puerto Rico: https://aldia.microjuris.com/2017/09/28/que-significa-la-dispensa-de-las-leyes-decabotaje-para-puerto-rico/

27 Debe añadirse aquí que en el derecho norteamericano existe una tensión añadida entre las municipalidades y los gobiernos centrales. En efecto, las entidades que solicitan acogerse al capítulo IX mantienen durante el procedimiento concursal su autoridad y su capacidad para decidir su política fiscal. Sin embargo, la decisión de acogerse a un procedimiento concursal en el marco del referido capítulo es una opción que no está exenta de consecuencias sobre otras municipalidades o, incluso, sobre otras unidades políticas de gobierno. Ello hace que los gobiernos centrales se planteen la opción del rescate en detrimento del concurso, lo que permite al Gobierno central imponer fuertes restricciones y condiciones a la municipalidad rescatada. Por el contrario, la municipalidad insolvente se encuentra con una herramienta que le permite presionar y amenazar al Gobierno central la asunción de los costes del rescate. 
la puesta en marcha de ciertas medidas por parte del Estado: ayudas directas de este, reformas de impuestos...28.

Dejando a un lado la posibilidad de crear este Consejo de Supervisión, se propone también la posibilidad de crear los «Budget Stabilization Funds» («BSF»), instrumento fiscal que permite el ahorro en épocas de prosperidad para hacer uso de esos fondos en momentos de crisis $^{29}$. La práctica pone de manifiesto que, en aquellos lugares donde se ha hecho uso de este mecanismo, ha permitido afrontar las crisis en mejores condiciones.

En definitiva, de lo expuesto se infiere que en aquellos modelos en los que se ha extendido el concurso a las administraciones territoriales, se opta por plantear soluciones alternativas, al considerar que el concurso per se, aunque adaptado al tipo subjetivo, no se revela como un instrumento idóneo para solventar las situaciones de crisis. $\mathrm{Ni}$ el patrimonio es liquidable, ni los servicios pueden dejar de prestarse. Pero es que, además, el procedimiento concursal no soluciona los problemas intrínsecos a las municipalidades y su financiación.

Es cierto que es necesario proveer soluciones para las crisis de las municipalidades, algo extraordinariamente frecuente en estos momentos. Y no es menos cierto que el régimen ordinario concursal no resulta adecuado en absoluto para las municipalidades. Aun cuando todo esto es así, tampoco puede negarse que han de buscarse soluciones porque la mayor parte de estos entes no pueden desaparecer sin más.

Ahora bien, ni el régimen concursal estadounidense ni el proceso de reestructuración previsto en PROMESA son panacea alguna, y ello porque su finalidad y objetivo es limitado: la reestructuración de la deuda. Sin embargo, es preciso encontrar soluciones para afrontar la crisis de las corporaciones de derecho público.

Medidas de limitación del techo de gasto y de control pueden introducirse para evitar o, por lo menos, poder anticiparse a las situaciones de crisis de las instrumentalidades públicas, tal y como se ha hecho en otros sistemas. De hecho, en España, existe un mecanismo específico para solventar las crisis

28 En el año 2003, Pittsburgh atravesó una fuerte crisis: un déficit presupuestario de treinta y cinco millones y un endeudamiento de ochocientos setenta y nueve millones de dólares. Esta fue la única ciudad que en los años 2009-2010 carecía ya de déficit según los datos de The Philadelphia Reseach Iniciative. Por su parte, el estado de Carolina del Norte puso en marcha una Comisión de Gobierno Local que ha tenido un éxito notable también (véase O. Kimhi, 20 Iо: 54-57).

29 Y. Hou (2006), «Budgesting for Fiscal Stability over the Business Cicle: a Countercyclical Fiscal Policy and the Multiyear Perspective on Budgeting», en Public Administration Review, 66, págs. 736-739 (pág. 730). 
financieras de los entes públicos: la Ley Orgánica de Estabilidad Presupuestaria y Sostenibilidad — Ley Orgánica 2/2012, de 27 de abril—, que desarrolla el nuevo art. 135 de la Constitución introducido por la reforma operada el 27 de septiembre de 2011. Estas reformas zanjan cualquier posible discusión que pudiera plantearse en relación con esta cuestión en tanto no se modifiquen los Tratados europeos de los que traen causa ${ }^{30}$.

Y no es que las administraciones territoriales españolas no se hayan encontrado nunca en situación de insolvencia. Más bien podemos decir que ha sucedido al contrario. A lo largo de 2012, diversas comunidades autónomas han ido extendiendo la mano y pidiendo ayuda al Estado, abandonando las fórmulas diseñadas al principio (la emisión de deuda en forma de «bonos patrióticos») para someterse y solicitar su «rescate» a través del Fondo de Rescate Autonómico creado por el Ministerio de Hacienda ${ }^{31}$, al que se han acogido diversas comunidades autónomas ${ }^{32}$. Hoy, las comunidades autónomas están sometidas a un fuerte control presupuestario y de gasto por parte del Ministerio de Administraciones Públicas, que controla su gasto y limita los flujos de caja cuando el déficit se dispara.

En el ámbito del derecho comparado se han seguido fórmulas diversas, aun cuando el planteamiento general es el de la no concursalidad de los entes

30 En concreto, el artículo 126 del Tratado de Funcionamiento de la Unión Europea DOUE núm. 83, de 30 de marzo de 2010- (en adelante TFUE) y el artículo 5 del Tratado de Estabilidad, Coordinación y Gobernanza (TECG) en la Unión Económica y Monetaria (2012).

31 Real Decreto Ley 21/2012, de 13 de julio, de medidas de liquidez de las administraciones públicas y en el ámbito financiero.

32 La Comunidad Valenciana fue la primera en acogerse al Fondo el 20 de julio de 2012, solicitando cuatro mil quinientos millones; Andalucía, al mismo tiempo que aprobó un techo de endeudamiento para 2013, el 8 de octubre de 2012, solicitó cuatro mil novecientos seis millones al Fondo; Castilla-La Mancha solicitó el 27 de septiembre de 2012, ochocientos cuarenta y ocho millones al Gobierno central con el que prevé abordar el $10 \%$ de la deuda financiera que acumula; Murcia, la segunda región en someterse al Fondo, pidió el 23 de julio trescientos millones, aumentando su solicitud a quinientos veintisiete millones quinientos mil el 28 de septiembre (comunidad autónoma rescatada que, a su vez, avaló a Aeromur para un préstamo de doscientos millones concedido por un holding bancario para terminar las obras del aeropuerto de Corvera); el 19 de octubre de 2012, Asturias y Baleares solicitaron un rescate por valor de doscientos sesenta y un millones, setecientos mil y trescientos cincuenta y cinco millones respectivamente; $\mathrm{Ca}$ taluña, una de las regiones con mayor PIB, fue la primera comunidad en recibir dinero del Fondo, ascendiendo su rescate a cinco mil trescientos setenta millones; finalmente, las islas Canarias se acogieron al Fondo el 5 de octubre de 2012, pidiendo setecientos cincuenta y otro millones ochocientos mil euros. 
públicos, al entender que estos prestan un servicio público y que, en consecuencia, no se puede prescindir de ellos. Con todo, debe reseñarse que en el ámbito europeo se constata no solo la falta de homogeneidad de los ordenamientos, sino también un cambio de enfoque o incluso de paradigma. En efecto, los procedimientos concursales se revelan inadecuados para atender las crisis de las personas jurídicas; generalmente, estos acaban en un proceso de liquidación por imposibilidad de reflotar la entidad. Ello ha llevado paulatinamente a optar por tratar de encontrar mecanismos que permitan reestructurar la deuda. Esta es la vía que se ha seguido, además, a nivel europeo, tal y como refleja la propuesta del 22 de noviembre de 2016. En efecto, la Comisión Europea publicó en la referida fecha el Proyecto de Directiva sobre reestructuración y segunda oportunidad ${ }^{33}$. Esta no es sino la continuación natural de la recomendación sobre un nuevo enfoque frente a la insolvencia y el fracaso empresarial $^{34}$ de 2014 , y se justifica por la necesidad de remediar un déficit normativo en los derechos nacionales de los Estados miembros en este ámbito. En Europa, hasta ahora, las soluciones venían de los legisladores nacionales. A diferencia de lo que sucede en los Estados Unidos, donde el derecho concursal es derecho federal (i.e. uniforme), en la Unión Europea el derecho concursal es estatal. Por consiguiente, el derecho interno de cada Estado miembro había abordado los problemas que plantean las empresas en situaciones de insolvencia o próximas a ella según sus propios principios y criterios. La consecuencia que se deriva del modelo es que el eje principal de la propuesta gira en torno a los acuerdos de reestructuración, inspirados claramente en el capítulo 11 norteamericano (aunque el procedimiento europeo es más flexible e informal). Habrá que esperar, pues, no solo a ver cómo queda finalmente diseñado el procedimiento, sino que habrá que esperar todavía más para comprobar si los efectos deseados son alcanzados.

33 https:/ec.europa.eu/transparency/regdoc/rep/1/2016/ES/COM-2016-853-F1-ESMAIN-PART-1.PDF

34 http://eur-lex.europa.eu/LexUriServ/LexUriServ.do?uri=OJ:L:2014:074:0065:0070: ES:PDF 
